\title{
Short History of Nuclear Many-Body Problem
}

\author{
H. S. Köhler ${ }^{\mathrm{a}}$ \\ ${ }^{a}$ Physics Department, University of Arizona, Tucson, Arizona 85721
}

\begin{abstract}
This is a very short presentation regarding developments in the theory of nuclear many-body problems, as seen and experienced by the author during the past 60 years with particular emphasis on the contributions of Gerry Brown and his research-group. Much of his work was based on Brueckner's formulation of the nuclear many-body problem. It is reviewed briefly together with the Moszkowski-Scott separation method that was an important part of his early work. The core-polarisation and his work related to effective interactions in general are also addressed.
\end{abstract}

\section{Dedication}

The main content of this work was presented at the Gerry Brown memorial conference in Stony Brook. I first met Gerry at least 55 years ago while he was with Rudolf Peierls in Birmingham and I was a graduate student in Uppsala and at CERN. I never worked directly with him (although he tried to recruit me for some of his projects). But I did interact with him in various ways over the years. In 1959 he was my opponent at my $\mathrm{PhD}$ thesis 1 defense in Uppsala (Sweden). Last time we met was at Eyvind Osnes retirement conference in Oslo in 2008. He told me then, after my talk, that I should have "spruced it up" like he himself does. He was a good friend. Gerry had many collaborators not only among his many students. He was always able to make others interested in problems he considered important. It was one of his strengths. That together with his enthusiasm, physical insight and intuitive thinking is how he will be remembered. His contributions to the problems of nuclear physics were dominating and will be ever-lasting. It

\footnotetext{
${ }^{1}$ On optical model with spin-orbit coupling
} 
is not possible to cover more than a small fraction of his work on many-body physics in this short talk. 


\section{Introduction}

I find the history of physics (almost) as fascinating as physics itself. A historical perspective shows a scenario of ideas and people behind the ideas often not found in published papers. The real physics is of course found in what experiments reveal to us. The human brain seeks to understand these physical phenomena and that is what theorists are trying. It is a fascinating interaction between us and the world around us. Theory is the subject of this presentation.

When does the history of the nuclear many-body problem start? One of the great discoveries was the nuclear shell-model. Liquid drop, collectivity, was the predominant and succesful picture theorists had before that. So how can one explain the success of the seemingly contradictive picture that the shell-model presented? Another problem: nuclear saturation. A possible explanation: N-N interactions are repulsive at short distances. [1]. But how can one reconcile the strong interactions with a shell-model? How can one deal with the strong and even infinitely repulsive forces computationally? These were some of the nuclear physics problems some 60 years ago.

The stage was set for someone to come up with a many-body theory of nuclear structure. The first successful nuclear many-body theory was that of K.A. Brueckner's. Gerry Brown's (and others) nuclear strucure work was based on this theory.

In the theoretical treatment of the nuclear many-body problem, nuclei are in general considered to be composed of nucleons (protons and neutrons) interacting with some specified forces without internal degrees of freedom. The solution of this problem is hampered by two difficulties.

I. The strength and complexity of interaction(s) that are also unknown in details.

II. The mathematics to solve for the physical properties of a many-body system.

The first problem (I.) is still being worked on in several ways. A method to overcome the second problem (II.) was presented by K.A. Brueckner some 60 years ago. It showed how the strong interaction can be replaced by an 'effective' softer interaction, more manageable to handle. The invention of the shell-model led to a very active research to interprete the experiments on nuclear spectroscopy. A crucial part of this work was of course then the choice of NN-interaction. Gerry Brown, understanding the significance of Brueckner's work, commenced very successful shell-model calculations together with 
collaborators using the Brueckner 'effective' interaction, the reaction-matrix, to do the job.

\section{Brueckner theory}

Related to the shell-model is the optical model of the 50's, that pictures nucleons moving in a mean field. It was explained by Francis and Watson as a multiple scattering problem with elementary scatterings being via 'soft' T-matrices rather than 'hard' N-N bare interactions. 2] This idea was picked up by Brueckner: Maybe a nuclear many-body theory for bound states could also be built on T-matrices instead of the, at the time, conventional efforts using NN-interactions with slowly converging or diverging results. But the T-matrix is complex

$$
T=v+v \frac{1}{k^{2}-k^{\prime 2}+i \eta} T \sim e^{i \delta} \sin (\delta)
$$

with the $\sim$ indicating the complex diagonal element. For calculating the real binding energies of nuclei it seemed to make some sense to replace this complex $T$-matrix with the real Reactance matrix (the R-matrix) defined by a principal value integration. [3]

$$
R \sim \tan (\delta)
$$

replacing the bare interaction with an 'effective' interaction

$$
V(k) \sim \tan (\delta(k))
$$

This idea had some degree of success when calculating nuclear bindings. BUT, the R-matrix refers to a scattering problem with boundary problems different from that of a bound state. It is fairly easy to show that if putting two interacting particles in a box, square or Harmonic oscillator (Busch formula) the binding energy is not $\sim \tan (\delta)$ but rather just $\delta$. 2 In the scattering problem one deals with a continuum set of states but in the bound state problem with a discrete set of states. "Infinite" nuclear matter still implies

\footnotetext{
${ }^{2}$ The Busch formula expresses the binding energy of two nucleons in an oscillator well in terms of phase-shifts. I recently showed that the SHIFT in energy like for the square box is given by $\delta$
} 
a bound state problem. Summation over a discrete set of states no matter how dense is different from integration over a continuum. [5, 6, 6] 3 Brueckner later improved the theory to include medium effects. The $\delta$-(or phaseshift) approximation is however still good at low density and also for 'weak' interactions as well as for large angular momenta $l \geq 4$, when medium-effects can be neglected.

What about the medium, many-body effects? We deal with an assembly of nucleons, a fermion-system. When summing over intermediate states, the occupied states should therefore be excluded, modifying the $T$-matrix (11). Brueckner together with Wada defined this modified effective interaction, the reaction matrix $K$ [8] to get

$$
K=v+v \frac{Q}{k^{2}-k^{\prime 2}} K
$$

with $Q$ being a Pauli-operator. Note that $K$ is real. No summation (integration) over a pole. No longer a discrete-continuum controversy. But the shell-model says that the nucleons move in a mean field $U(k)$. Accordingly energies are not $e(k)=k^{2}$ but rather $e(k)=k^{2}+U(k)$.

This additional modification results in what is usually referred to as Brueckner theory, summarised as follows. Brueckner Reaction Matrix $K$ defined by [9]:

$$
K=v \frac{Q}{e(k)-e\left(k^{\prime}\right)} K
$$

Total energy (first order):

$$
E_{T}=\sum k^{2}+\frac{1}{2} \sum K
$$

Mean field:

$$
U(k)=\sum K
$$

This equation for $U(k)$ is often referred to as a Brueckner self-consistency because $U$ is a functional of $K$.

The Brueckner $K$-matrix sums ladder and mean-field propagations to all orders. Infinite nuclear matter calculations show saturation and binding

\footnotetext{
${ }^{3}$ The difference between $\delta$ and $\tan (\delta)$ may of course be negligible for small $\delta$. With large scattering lengths especially with $\delta=\frac{\pi}{2}$ it makes a big difference.
} 


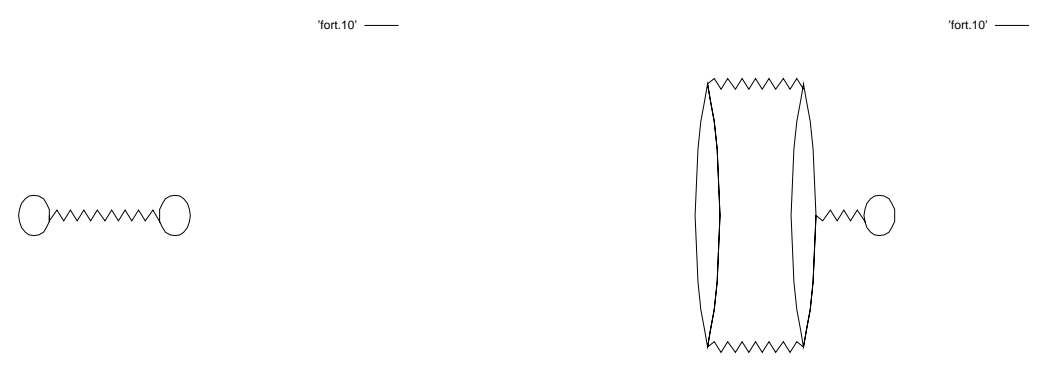

Figure 1: Diagrams included in the Brueckner $K$-matrix approximation. The diagram to the right is a three-body term. The interaction between two particles is influenced by the presence of a third, the tadpole. This diagram relates to the dispersion-term that is an important term in the separation-method. See section below.

energy in remarkable agreement with experimental information. Important physics is included in this first order in $K$ approximation. Improved results can (in principle) be obtained by higher orders but convergence problems are not well understood.[10, 11]

It is a zero spectral-width approximation. Spectral widths can be included in higher orders or Green's function calculations but showing relatively small corrections.

Gerry Brown (with co-workers) had several publications related to nuclear matter. In a 1964 paper, Gerry Brown and coworkers investigated the effect of various approximations and off-energy shell propagations.[12] In a 1982 paper with Hans Bethe the focus was on compressibility an important matter in relation to supernovaae explosions. [13]

\section{Separation Method}

Much of the computational work on Brueckner theory in the early 60's was to find suitable approximations as the access to computers at that time was limited. The equations are nowadays very simply solved numerically. The separation method introduced by Moszkowski and Scott [14] for evaluating the reaction matrix proved valuable not only as a computational tool but it also gives valuable insight into the physics. It also relates to more recent ideas behind EFT and $V_{\text {low- }}$ as presented in a section below. The method involves dividing the nucleon-nucleon potential $V$ into two parts in ccordinate space, 
the short-ranged part $V_{s}$ and the long-ranged part $V_{L} . V_{s}$ will have a repulsive as well as an attractive part. A separation distance $d$ (in coordinate space) is chosen such that the scattering phase-shift for the potential $V_{s}$ is zero. This is of course momentum-dependent so that $d=d(k)$. A slight modification of the method was made by Köhler [15]. He obtained (in operator-form)

$$
K=K_{s}+V_{L}+(\Omega-1) e(Q-1)(\Omega-1)+(\Omega-1) U(\Omega-1)+2 V_{L}\left(\frac{Q}{e}\right) V_{L}
$$

$\Omega$ is the wave-operator for the short-ranged part therefore related to correlations. Of particular significance is the fourth term proportional to the mean field $U(k)$ as well as the correlation-volume $I_{D}$ (the "wound-integral").

$$
I_{D}=\int(\psi-1)^{2} d \mathbf{r}
$$

where $\psi$ is the in-medium NN-correlated wave-function. (See Fig. (3) below).

This fourth term is referred to as the dispersion term.

$$
K_{d i s p} \sim I_{D} * U
$$

It is repulsive and important for providing nuclear saturation. It is a 3-body term with two particles interacting with other particles in the medium which is represented by the momentum-dependent mean field $U(k)$. It involves offshell scattering and therefore model-dependent, while the on-shell scattering is fixed by fits to free space scattering. The separation method was used by Gerry Brown and co-workers (and others) in shell-model calculations.

\section{Core-polarisation}

While the Brueckner reaction-matrix gives an effective interaction based on 'realistic' forces between the constituent nucleons, results of early shellmodel calculations were not promising. Gerry Brown realised that an important contribution to the interaction, beyond the reaction-matrix, was missing. The clue to the problem is that particle-hole excitations are allowed in the finite system. This results in an additional term, the core-polarisation shown diagramatically in Fig. (2)

It was first included in calculations by George Bertsch [16] for ${ }^{18} \mathrm{O}$ and ${ }^{42} S c$ using the Kallio-Kolltveidt force. [17] This paper was followed by the seminal papers by Tom Kuo and Gerry Brown on the "Structure of Finite 


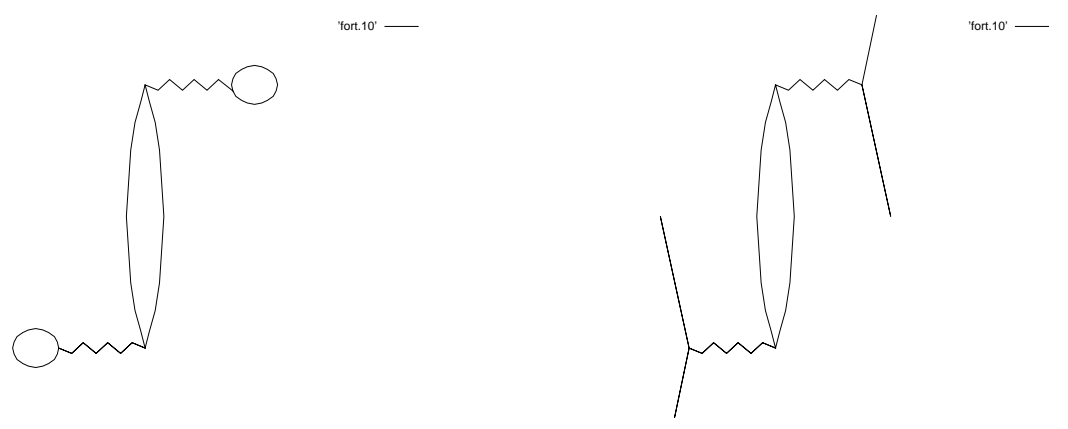

Figure 2: Lowest order core-polarisation diagrams. To the left is the energy-diagram. Breaking the two loops results in the core-polarisation diagram for the effective interaction as shown to the right. The energy diagram is cancelled by a proper choice of the mean field .

Nuclei and the Free Nucleon-Nucleon Interaction" [18, 19, 20]. Here the reaction matrix as well as the core-polarisation was calculated from the HamadaJohnston potential[21] using the separation method. The Kuo-Brown interaction has been, and still is used by many research-groups in nuclear structure calculations. 4 Barrett and Kirson questioned the convergence of the corepolarisation diagrams. 225] In a paper by Anastasio et al the effect of the choice of shell-model mean field potential is illustrated. [26]

\section{6. $V_{\text {low }-k}$}

A many-body problem is always a two-part problem:

1. Interactions between particles e.g. 2-,3- etc interaction potentials.

2. A many-body theory.

The theory of nuclear forces has been a long-standing problem. (see e.g. ref. [23]). It is easy to construct NN-potentials that fit scattering phase-shifts as well as deuteron data e.g. by inverse scattering and separable potentials. 24] But that is in general not enough. Off-shell scattering information is needed for many-body calculations. This was for instance already emphasized by Gerry Brown and coworkers in the 1964 paper. [12] It was al-

\footnotetext{
${ }^{4} \mathrm{~A}$ more complete summary and history of these and following works are found in the book by Gerry Brown and coworkers. 22.
} 
ready stated above that the dispersion effect depends on off-shell scattering. This is actually a main problem in nuclear many-body calculations. That is why we need a theory of nuclear forces going beyond the on-shell information obtained from scattering. And off-shell scattering is directly related to correlations, short-ranged as well as tensor-induced.

It can however also be argued that the high energy component of the interaction (i.e. the largely un-known short-ranged part) should be less relevant in dealing with low-energy nuclear problems. The low and high energy components are separated when using the separation method shown above with $V_{L}$, the long-ranged part, being the leading term.

A different approach whereby the high energy component of the force was eliminated by integrating out the largely unknown and model dependent part of the interaction was presented by Bogner et al [27, 28]. The resulting low-momentum potential is referred to as $V_{l o w-k}$ and it is a function of the chosen cut-off momentum $\Lambda$. This cut-off has a motivation similar to the cut-off in coordinate space in the separation method. The near numerical equivalence was shown in a paper by J. W. Holt and G. E. Brown. 29]

A more fundamental approach to the separation of momentum scales is EFT (Effective Field Theory) originated by Weinberg. [30] Not suprisingly, Gerry Brown was consulted by Weinberg.

Correlations in nuclear matter are however important. This is explicitly illustrated in the separation method where the wound-integral (9) is a measure of the correlations. The dispersion term (10), being a product of the mean field and the wound-integral, is responsible for saturation and nuclear stability. A too small momentum cut-off quenches the correlations so that

$K_{\text {disp }} \rightarrow 0$ with no saturation. [31] The situation is illustrated by the two set of curves in fig (3) . Fig. (44) shows the influence of the loss of correlations (and the dispersion-effect) on the binding energy. It should also be noted that the dispersion effect is much smaller in the singlet channel in agreement with Fig. (3). These are results of standard Brueckner calculations at normal nuclear matter density.

\section{Summary and Future}

The last fifty years of developing methods to solve the nuclear manybody problems have been an exciting time. Keith Brueckner's pioneering work and Gerry Brown's application of Brueckner's method to the nuclear shell-model problem will remain as corner-stones in the history of nuclear 

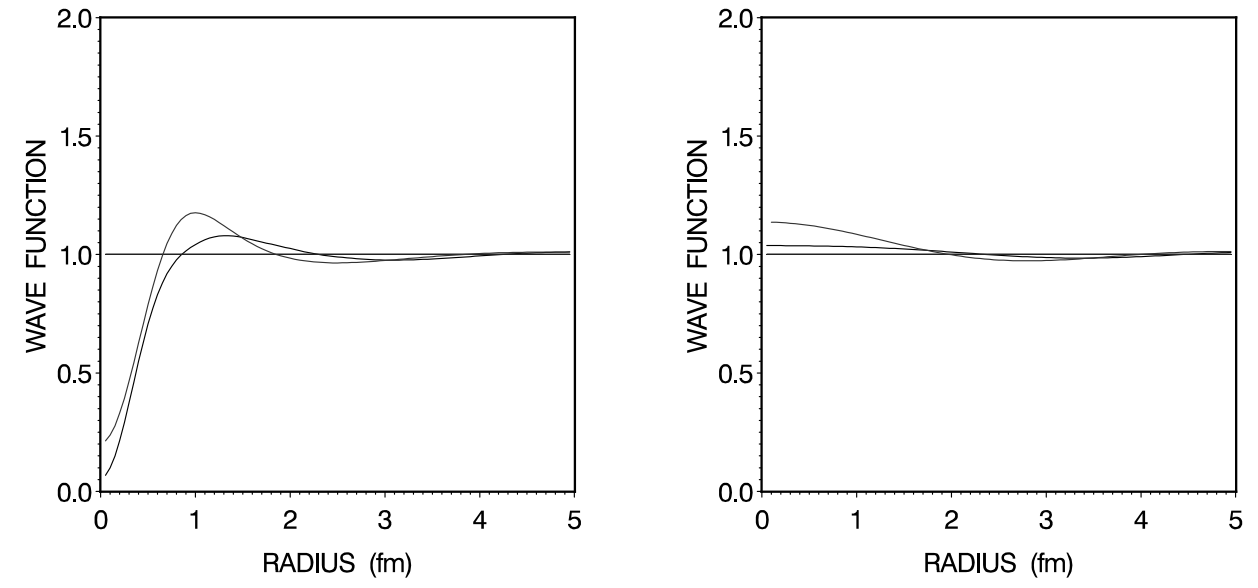

Figure 3: Left curves:The correlated wave functions for triplet (upper curve) and for singlet interactions (lower curve) with a cut-off $\Lambda=9.8 \mathrm{fm}^{-1}$. Right curves: Similar to the left curves but for a cut-off $\Lambda=2 \mathrm{fm}^{-1}$.

theory. The outcome of the numerical work relates of course closely to the (free) nucleon-nucleon interactions. These are in particular complicated in relation to induced correlations. The last few years have shed important light on this problem assuming that the low energy nuclear problem should not be impacted (in a major role) by short-ranged (high energy) components of the forces. Much care has however to be taken in order not to loose important effects of tensor and short-ranged effects. This was illustrated above and the relation to the dispersion term in the separation method expansion was emphasized. This is a three-body term and has effects similar to that of three-body forces but with separate physical content.

The nuclear many-body methods have been of significance not only for developments in nuclear theory, but also for developments in atomic and molecular physics as well. It has also inspired researchers to develop competing methods such as coupled cluster, hypernetted chain, Green's function methods to name a few. The density functional methods should also be men- 


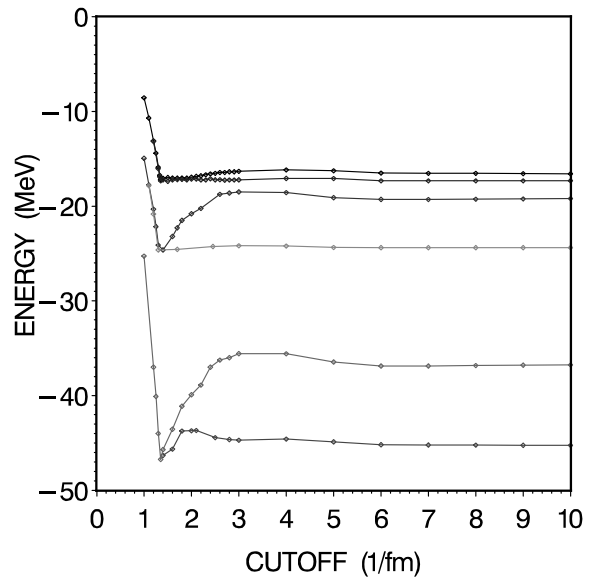

Figure 4: The potential energy per particle is shown as a function of cut-off $\Lambda$. The three set of curves show from top to bottom: singlet S-state, triplet S-state and total contributions (all angular momenta). At each set there are two curves: The lower is without the mean field (i.e. without dispersion effect) and the upper is with mean field, thus with less attractive effective force. The loss of correlations shows binding to increase dramatically for $\Lambda \leq 2.5$. Note also the difference in dispersion effect between the singlet and triplet states. This agrees with the difference in correlations in the two cases as shown in Fig. (3).

tioned in this context. All of the improvements in applying these methods have of course followed the improvements in computer capabilities and facilities. 5 These have in particular benefitted the Monte-Carlo and shell-model (in particular the no-core shell-model) calculations.

In astrophysics and heavy-ion collision problems one deals with nuclear matter with densities well beyond those of the nuclear saturation point, that are not reachable by Brueckner or similar methods. There are many efforts

\footnotetext{
${ }^{5}$ The author feels compelled to remind of the difficulties in 1980's when he and others had to 'escape' to Europe where such facilities were more readily available
} 
of extrapolation by compressibility calculations with these methods. The Rho-Brown scaling procedure is one approach to this problem. [32] There is evidence that 3-body forces are important in nuclear structure calculations. At higher densities 4-body (and higher) forces are likely to come into play. Brueckner theory, as usually practiced, includes medium-effects up to 3-body terms. At higher densities higher order terms (diagrams) become increasingly important. This is a challenging problem for theorists.

\section{References}

[1] Robert Jastrow, Phys. Rev. 81 (1951) 165.

[2] N.C. Francis and K.M. Watson, Phys. Rev. 92 (1953) 291.

[3] Brueckner, Levinson and Mahmoud, Phys. Rev. 95 (1954) 217.

[4] Alfred Reifman and Bruce S. DeWitt Phys. Rev. 101 (1956) 877.

[5] N. Fukuda and R.G. Newton Phys. Rev. 103 (1956) 1558.

[6] B.S. DeWitt Phys. Rev. 103 (1956) 1565.

[7] W.B. Riesenfeld and K.M. Watson Phys. Rev. 104 (1956) 492.

[8] K.A. Brueckner and W. Wada, Phys. Rev. 103 (1956) 1008.

[9] K.A. Brueckner and J. L. Gammel, Phys. Rev. 109 (1958) 1023.

[10] H. A. Bethe, Annual Review of Nuclear Science 21 (1971) 93.

[11] H.S. Köhler, Physics Reports 18C (1975) 217.

[12] G. E. Brown, G.T. Schappert and C.W. Wong, Nucl. Phys. 56 (1964) 191.

[13] H. A. Bethe, G. E. Brown, J. Cooperstein and James R. Wilson, Nucl. Phys. A 403 (1983) 625.

[14] S.A. Moszkowski and B.L. Scott, Annals of Physics 11 (1960) 65.

[15] H.S. Köhler, Annals of Physics 16 (1961) 375.

[16] George F. Bertsch, Nucl. Phys. 74 (1965) 234. 
[17] A. Kallio and K. Kolltveidt, Nucl. Phys. 53 (1964) 87.

[18] T.T.S. Kuo and G.E. Brown, Nucl. Phys. 85 (1966) 40.

[19] T.T.S. Kuo and G.E. Brown, Nucl. Phys. A 92 (1967) 481.

[20] T.T.S. Kuo and G.E. Brown, Nucl. Phys. A 114 (1968) 241.

[21] T. Hamada and T. D. Johnston, Nucl. Phys. 34 (1962) 383.

[22] Gerald E. Brown,Thomas T. Kuo, Jeremy W. Holt and Sabine Lee, World Scientific Publishing Co (2010)

[23] R. Machleidt, Advances in Nuclear Physics 19 (1989) 189, Plenum Press edited by J.W. Negele and Erich Vogt.

[24] N. H. Kwong and H. S. Köhler, Phys. Rev. C 55 (1997) 1650.

[25] B. R. Barrett and M.W. Kirson, Nucl. Phys. A 148 (1970) 145.

[26] M. R. Anastasio, T.T.S. Kuo, T. Engeland and E. Osnes, Nucl. Phys. A 271 (1976) 109.

[27] Scott Bogner, T.T.S. Kuo, L.Coraggio, Nucl. Phys. A 684 432(c) 2001

[28] Scott Bogner, T.T.S. Kuo, L.Coraggio, A.Covello and N. Itaco Phys. Rev. C 65 051301(R) 2002

[29] J. W. Holt and G. E. Brown, arxiv:nucl-th/0408047

[30] Steven Weinberg, Phys. Lett. B 251 (1990) 288.

[31] H. S. Köhler and S.A. Moszkowski, arxiv:nucl-th/0703093

[32] G. E. Brown and Mannque Rho, Physics Reports 396 (2004) 1. 